\title{
Assessment of the seismic capacity of stone masonry walls with block models
}

\author{
José V. Lemos, A. Campos Costa and E. M. Bretas \\ LNEC - Laboratório Nacional de Engenharia Civil \\ Av. do Brasil 101, 1700-066 Lisboa, Portugal \\ vlemos@1nec.pt, alf@lnec.pt, ebretas@lnec.pt
}

\begin{abstract}
The application of discrete element models based on rigid block formulations to the analysis of masonry walls under horizontal out-of-plane loading is discussed. The problems raised by the representation of an irregular fabric by a simplified block pattern are addressed. Two procedures for creating irregular block systems are presented, one using Voronoi polygons, the other based on a bed and cross joint structure with random deviations. A test problem provides a comparison of various regular and random block patterns, showing their influence on the failure loads. The estimation of natural frequencies of rigid block models, and its application to static pushover analyses, is addressed. An example of application of a rigid block model to a wall capacity problem is presented.
\end{abstract}

Keywords: Masonry structures, seismic assessment, discrete elements, rigid blocks. 



\section{INTRODUCTION}

The safety assessment of historical masonry structures under seismic loads requires numerical models with the ability to represent the types of failure modes observed in earthquakes. Laboratory tests provide a controlled environment in which the behavior can be more completely characterized. Block models, based on the discrete element method, are one of the numerical tools available to simulate phenomena such as sliding and separation along joints, which lead to progressive structural damage and collapse. Their application to structural components or monuments of a relatively small size, for which the individual blocks can be numerically represented, poses no major difficulties [1]. The success of this type of application has encouraged the extension of these models to more complex structures, for example, involving masonry walls formed by irregular blocks, or multiple leaf walls, as found in many historical constructions. In these cases, the numerical idealization requires much more drastic simplifications that need to be critically assessed.

The present work addresses the application of rigid block models to analyze the out-of-plane behavior of masonry walls under horizontal loads. The influence of the block patterns on the results is discussed. Block systems based on a simple idealized geometric pattern, typically used in many applications, are compared with randomly generated block systems, using two different procedures. The first is based on Voronoi polygons, which may be representative for some types of masonry, but does not reproduce the laying in courses. The second method is a new proposal based on a bed joint and cross joint structure, but with some degree of randomness applied to joint spacing and orientation.

In these comparative analyses, the seismic action is represented simply as a static horizontal load, which makes the differences in behavior more evident. It should be remarked, however, that the advantages of rigid block models are more significant in dynamic analysis with explicit algorithms, because of the lower run times in comparison with deformable block models. The evaluation of natural frequencies is essential to a correct understanding of the seismic behavior. This may also be done with a rigid block model, by assuming an elastic behavior of all contacts. Since in these models, the system deformability is expressed in terms of joint (or contact) stiffnesses, for which experimental values are often lacking, the knowledge of eigenvalues and eigenmodes is very helpful in calibrating the model parameters, particularly when ambient noise measurements are available, which characterize the global dynamic response in the low level range. A straightforward procedure to calculate natural frequencies for a rigid block system is discussed. An application to the evaluation of the ultimate capacity of a large wall in a historical building is finally presented. 


\section{RIGID BLOCK MODELLING OF MASONRY WALLS}

Discrete element models employing polyhedral rigid blocks have proved very effective in the dynamic analysis of structures and monuments composed of blocks of hard rock with dry joints. Classical column-architrave structures are a typical case in which the numerical model may reproduce the individual blocks with reasonable accuracy [2]. The actual position and geometry of each block can be represented, even the existence of broken or damaged blocks.

Modern brick walls, for which unit shapes are known, but not their precise location, may be analyzed with either discontinuum or homogenized continuum models [3]. For large structures, the latter are more straightforward and less time consuming, even if failure modes are more rigorously simulated with the former.

The analysis of a wall formed by coursed or irregular masonry with mortared joints as a continuum appears more natural, since in practice the actual block geometry is not known. A discrete block model of such a wall is necessarily a simplified representation intended to follow the block pattern, not the exact shapes. The advantages of discrete element models for analyzing failure modes, always involving separation of the wall into blocks, have encouraged research in this area. Several approaches have been attempted, resorting to various levels of geometrical and mechanical complexity. Casolo [4] adopts a very simple block pattern, with continuous orthogonal joints, with all the complexity of masonry behavior being accounted for by elaborate joint constitutive models. At the opposite end, bonded particle models [5] employ large random assemblies of particles to simulate the irregular masonry units and the mortar [6], while relatively simple contact laws are used. In this paper, an intermediate approach is adopted, with polyhedral block systems generated according to various geometrical schemes, involving both regular patterns and systems with various degrees of irregularity and randomness. The mechanical interaction between the blocks is represented by standard MohrCoulomb joint models. A similar type of model was employed by De Felice and Giannini [7] to investigate the effect of block size on the out-of-plane resistance of masonry walls.

In a rigid block model, all system deformation is represented by relative movements between the blocks. In the early stages of loading, before the nonelastic behavior becomes dominant, the joint normal and shear stiffness parameters govern the system deformation. Therefore, they must account for both the block deformation and the joint deformation, either in the case of mortared or dry joints. Experimental data on joint stiffness show significant scatter, therefore the global deformability of a rigid block model must always be checked and assessed, to ensure that it is realistic. As block shapes and sizes do not reproduce rigorously the real patterns, some calibration of the stiffness parameters is essential. For dynamic problems, natural frequencies in the linear range can be contrasted with field measurements, providing an important contribution to the model calibration procedure. The ability of rigid block systems, assuming elastic contacts, to supply 
natural frequencies has been verified by comparison with analytical continuum solutions for walls [8].

\section{ANALYSIS OF INFLUENCE OF BLOCK PATTERNS}

The influence of the joint patterns adopted for the rigid block representation of the wall was analyzed with a simple test problem (Fig. 1). The wall was assumed to be simply supported in the out-of-plane direction at both lateral ends, by means of 2 fixed blocks, representing the effect of cross walls. The wall dimensions are $20 \times 10 \mathrm{~m}$, with $0.80 \mathrm{~m}$ thickness. For simplicity, a Coulomb friction model was adopted for the joints, without cohesion or tensile strength. A Young's modulus of 2.5 GPa was assumed. The joint stiffnesses listed in Table 1 correspond to an average joint spacing of $1 \mathrm{~m}$. For other spacings, these values were scaled to maintain an average elastic isotropy. Static analyses were conducted by applying a horizontal mass force in the out-of-plane direction. This load was increased in steps until failure. The analyses were performed with the code 3DEC [9].

Table 1. Joint properties for test problem

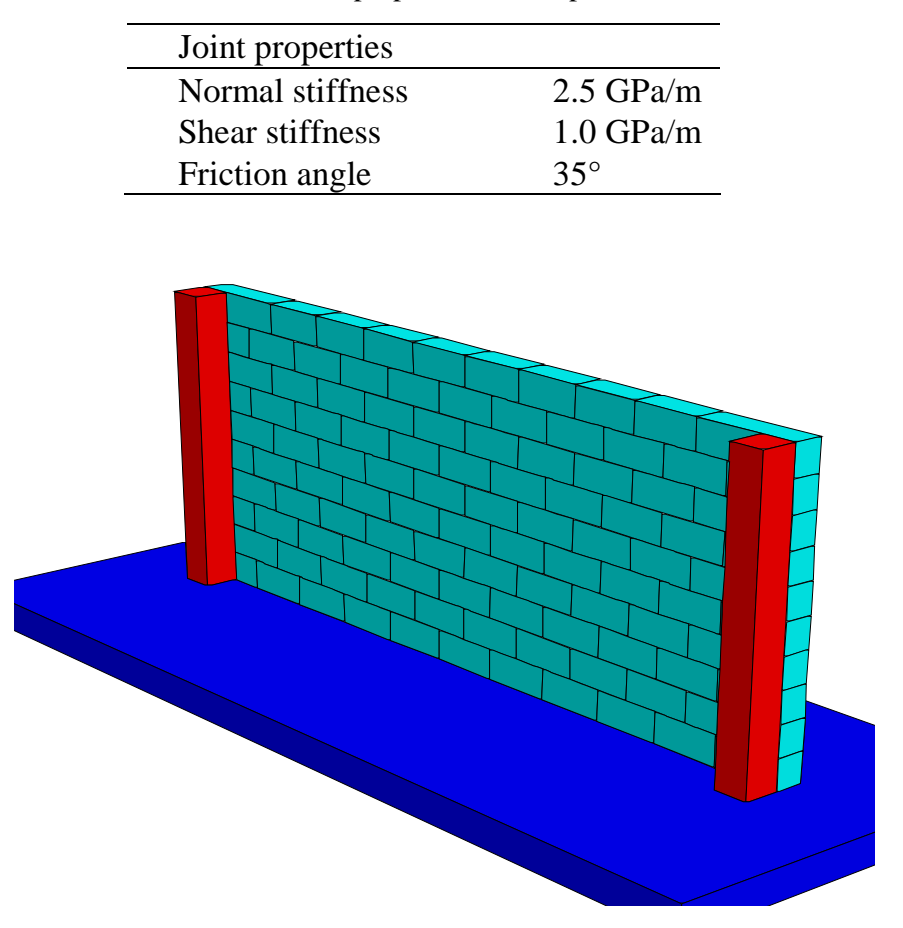

Fig. 1. Test problem (case of vertical joint offset of 1.0). 


\subsection{Regular block patterns}

The model shown in Fig. 1 corresponds to the case of blocks with dimension $2 \times 1 \mathrm{~m}$, with staggered vertical joints with an offset of $1.0 \mathrm{~m}$. For these block dimensions, 3 other cases were considered: continuous vertical joints (no offset), and staggered vertical joints with offsets of 0.5 and $0.1 \mathrm{~m}$. Fig. 2 illustrates the 4 block patterns analyzed.

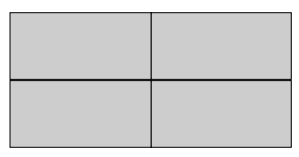

(a)

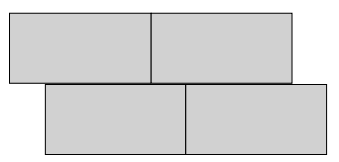

(c)

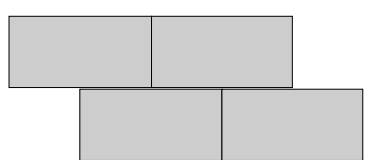

(b)

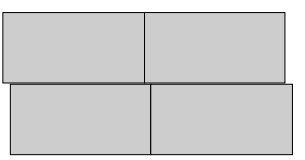

(d)

Fig. 2. Block patterns (block dimensions $2 \times 1 \mathrm{~m}$ ): (a) no offset; (b) offset=1.0; (c) offset=0.5; (d) offset $=0.1$.

A second series of tests were conducted with square blocks, dimensions of $1 \mathrm{x} 1$ $\mathrm{m}$. The 4 block patterns are illustrated in Fig. 3: continuous vertical joints, and 3 cases of discontinuous joints with offsets $0.5,0.25$ and $0.1 \mathrm{~m}$.

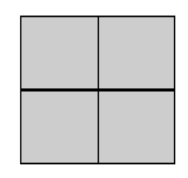

(a)

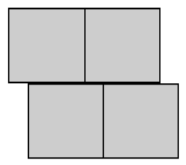

(c)

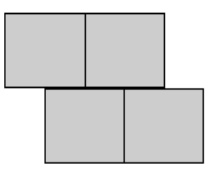

(b)

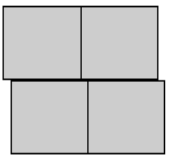

(d)

Fig. 3. Block patterns (block dimensions $1 \times 1 \mathrm{~m}$ ): (a) no offset; (b) offset=0.5; (c) offset=0.25; (d) offset $=0.1$. 
The results for the case of blocks with dimensions $2 \times 1 \mathrm{~m}$ are shown in Fig. 4a. The curves represent the out-of-plane displacement of the middle point at the top of the wall (horizontal axis) versus the horizontal gravity force (vertical axis). The horizontal force was incremented in steps of $0.1 \mathrm{~g}$, up to failure. The last point in each curve corresponds to the last equilibrated state. The corresponding curves for the case of blocks $1 \times 1 \mathrm{~m}$ are plotted on Fig. $4 \mathrm{~b}$.

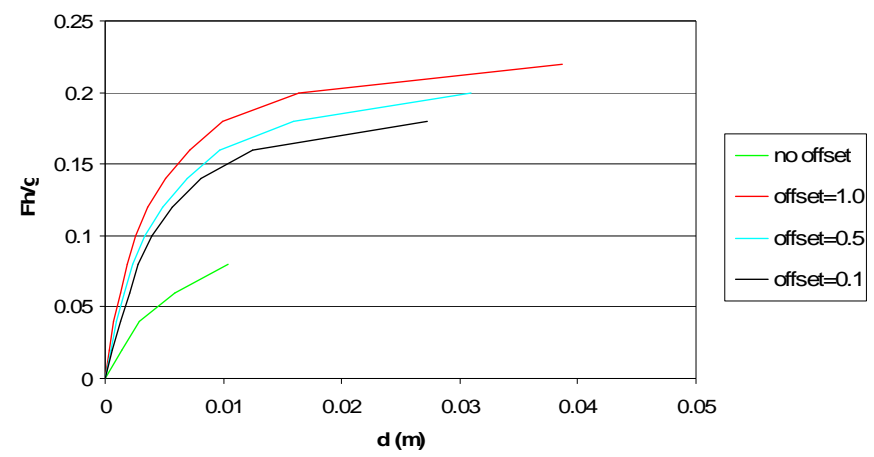

(a)

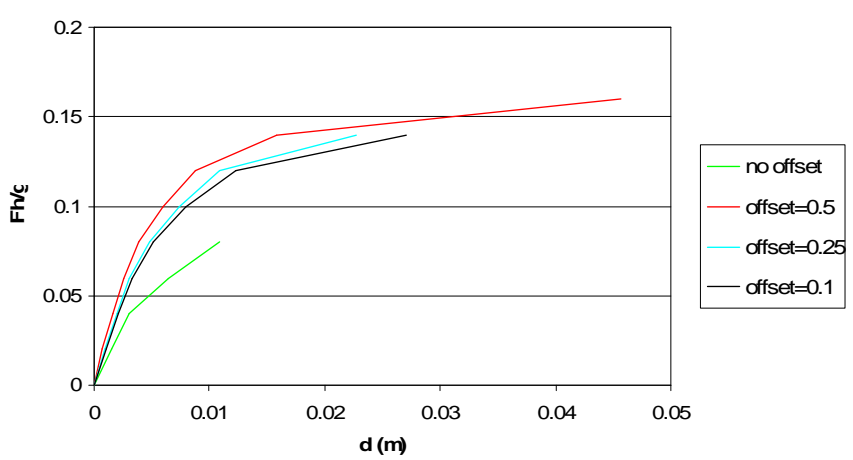

(b)

Fig. 4. Horizontal force vs. displacement curves for the 4 block patterns: (a) blocks $2 \times 1 \mathrm{~m}$; (b) blocks $1 \mathrm{x} 1 \mathrm{~m}$.

Considering first the case of rectangular blocks (Fig. 4a), it can be seen that the most significant difference is between the case of continuous vertical joints and the models with staggered joints. Even a small offset increases substantially the wall capacity. The chart for the case with square blocks (Fig. 4b) shows that the capacity of the wall with continuous joints is not altered (within the resolution of the load increment used). The staggered joint models display lower strength than 
those with rectangular blocks. This is related to the fact that the smaller areas of block contact along the horizontal joints lead to a reduced restraint of relative block rotation necessary to create the failure mode. These results show that the typical "brick wall" pattern often used as a representation of an irregular masonry wall may overestimate its strength. This is particularly significant, since numerical models often use larger block sizes than the real ones to save computational effort. Therefore the overestimation of the actual imbrication of the wall stones may adversely affect the safety assessment. Considering continuous joints is a rather conservative assumption, as offsets certainly exist, but may be defensible if the actual wall units are much smaller than the numerical blocks.

\subsection{Voronoi block patterns}

The numerical generation of block assemblies that represent correctly the various types of traditional masonry is a topic still demanding more research. Random shape patterns based on Voronoi polygons (or polyhedra) have been found to reproduce the geometries of some natural physical systems. For example, in rock mechanics, these shapes are now used to simulate the grain structure, defining the potential fracture paths [10]. Pina-Henriques and Lourenço [11] also used Voronoi patterns to study the fracture of masonry units in very detailed analyses at the meso-scale.

Herein, Voronoi patterns are used to create the random block pattern of an irregular masonry wall. These models are certainly not realistic for most types of masonry, which display block patterns where horizontal joints are more or less well defined, reflecting the way in which they were built. The block generator presented in the following section will address this point.

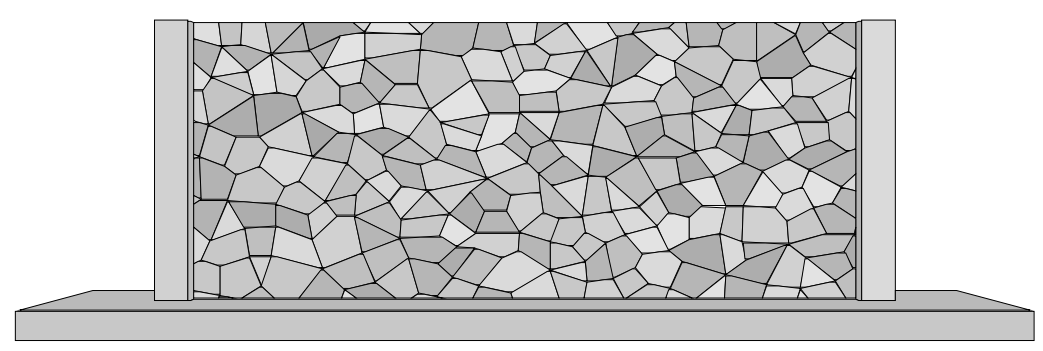

Fig. 5. Model with Voronoi block pattern (case 1). 
In this first study, the block pattern was obtained using a 2D Voronoi polygon generator. An average edge length of $1 \mathrm{~m}$ was assumed, to be comparable with the square blocks in the previous section. The 3DEC blocks were created assuming a uniform shape across the wall thickness. Fig. 5 shows one the several bock assemblies analyzed. Joint properties were the same as those in Table 1. The results of the simulations with 3 Voronoi block systems are compared in Fig. 6 with two of the square block models presented above (with continuous vertical joints and offset of $0.1 \mathrm{~m}$ ). First, it is interesting to note that the three randomly generated Voronoi patterns follow fairly similar deformation curves. The initial deformability of the system is close to that obtained with continuous vertical joints. However, the strength is higher, but still below the value obtained with imbricated joints with the smallest offset. The Voronoi pattern does not create discontinuous joints, so it tends to underestimate the block interlocking. The failure mode of the one of the Voronoi models is illustrated in Fig. 7.

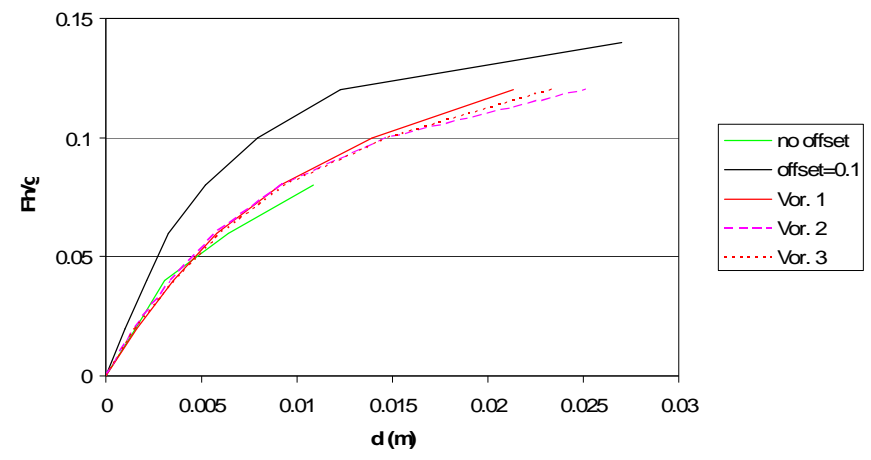

Fig. 6. Horizontal force vs. displacement curves for regular and Voronoi block patterns.

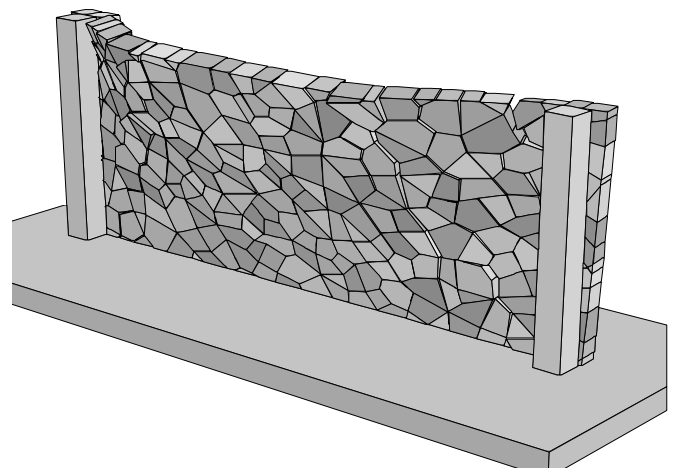


Fig. 7. Failure mode of model with Voronoi block pattern (case 1).

\subsection{A procedure for generation of irregular block patterns based on a bed joint and cross joint structure}

A block generation procedure for use in discrete element models of masonry was developed that is based on a typical bed joint and cross joint structure, but introduces some degree of randomness. In contrast with the Voronoi generator, this method produces a pattern of blocks arranged in courses with different heights and variable cross joint spacing. It is far from reproducing the complexity of masonry construction, but it is a useful tool to assess the influence of geometric variability on the deformation and failure processes.

The procedure starts by generation of continuous bed joints, each one formed by a set of segments (Fig. 8). The geometry of the bed joints defined by the following parameters: spacing $\left(\mathrm{s}_{\mathrm{m}}, \mathrm{s}_{\mathrm{d}}\right)$, segment length $\left(\mathrm{t}_{\mathrm{m}}, \mathrm{t}_{\mathrm{d}}\right)$, vertical deviation $\left(\mathrm{h}_{\mathrm{m}}\right.$, $h_{d}$ ) from mean trace. Each of these parameters is defined statistically in terms of a mean value $(\mathrm{m})$ and a deviation (d). In the present study a uniform distribution was assumed, for simplicity. For example, for the spacing of bed joints, a random number is generated in the interval $\left[\mathrm{s}_{\mathrm{m}}-\mathrm{s}_{\mathrm{d}}, \mathrm{s}_{\mathrm{m}}+\mathrm{s}_{\mathrm{d}}\right]$. However, more elaborate distributions may be employed. Cross joints are defining by their spacing $\left(b_{m}, b_{d}\right)$ and angle deviation from the vertical $\left(a_{m}, a_{d}\right)$.

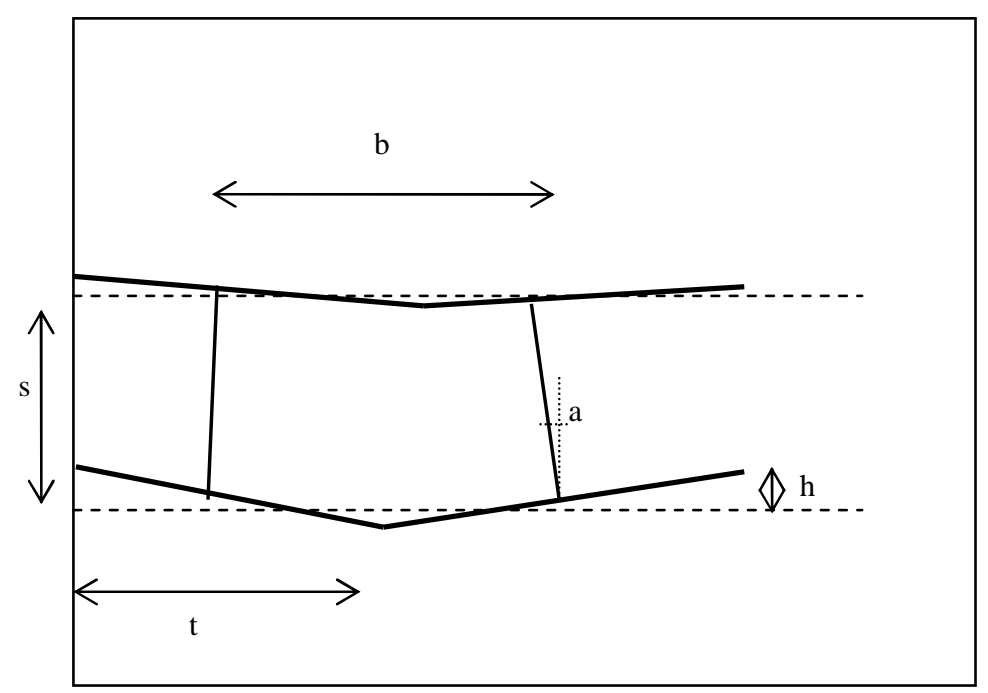

Fig. 8. Definition of geometric parameters in block generation procedure. 
The block generation procedure starts by creating the means traces of the bed joints, using the random spacing parameter. Then, each bed joint is created, composed of continuous segments, defined by their length and vertical deviation from mean trace. Finally, the cross joints are inserted, course by course, to form the block structure. This procedure produces blocks with slight concave angles, so an additional step may be taken, in which the concave blocks are split by extra cross joints. In this study, as the degree of irregularity was not large, the concave blocks were retained. Fig. 9 shows a system created with the parameters in Table 2.

Table 2. Parameters for irregular block generation

\begin{tabular}{|c|c|c|c|c|c|c|c|c|c|}
\hline $\begin{array}{c}\mathrm{s}_{\mathrm{m}} \\
(\mathrm{m})\end{array}$ & $\begin{array}{c}\mathrm{s}_{\mathrm{d}} \\
(\mathrm{m})\end{array}$ & $\begin{array}{c}\mathrm{t}_{\mathrm{m}} \\
(\mathrm{m})\end{array}$ & $\begin{array}{c}\mathrm{t}_{\mathrm{d}} \\
(\mathrm{m})\end{array}$ & $\begin{array}{c}\mathrm{h}_{\mathrm{m}} \\
(\mathrm{m})\end{array}$ & $\begin{array}{c}\mathrm{h}_{\mathrm{d}} \\
(\mathrm{m})\end{array}$ & $\begin{array}{c}\mathrm{b}_{\mathrm{m}} \\
(\mathrm{m})\end{array}$ & $\begin{array}{c}\mathrm{b}_{\mathrm{d}} \\
(\mathrm{m})\end{array}$ & $\begin{array}{c}\mathrm{a}_{\mathrm{m}} \\
\left({ }^{\circ}\right)\end{array}$ & $\begin{array}{c}\mathrm{a}_{\mathrm{d}} \\
\left({ }^{\circ}\right)\end{array}$ \\
\hline 1 & 0.1 & 2 & 1 & 0 & 0.2 & 1.5 & 0.5 & 0 & 0 \\
\hline
\end{tabular}

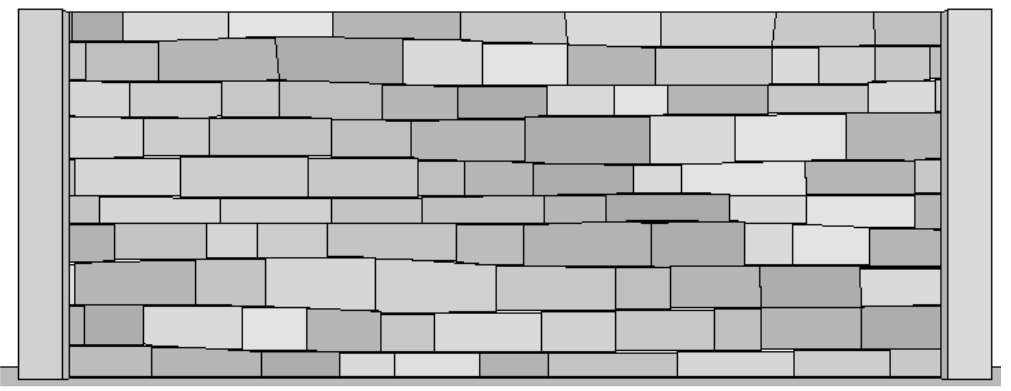

Fig. 9. Block system generated with parameters in Table 2.

The system in Fig. 9 is one of three randomly created with the geometric data listed in Table 2. The force-displacement curves obtained with these 3 models are compared in Fig. 10 with those from regular jointed models already presented (rectangular blocks). It may be seen that the 3 irregular patters display failure loads in the same range, which are also close to the case of the regular pattern with the smallest offset (0.1), and above the curve for the continuous cross joints. 


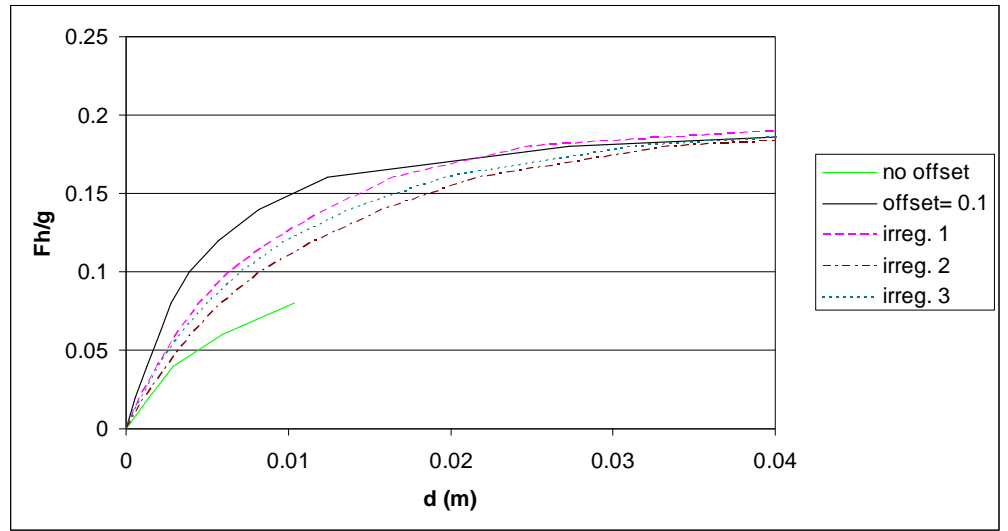

Fig. 10. Horizontal force vs. displacement curves for regular and irregular block patterns.

It should be noted that in this type of model the mortar is not represented, so the stone blocks are extended to include half of the joint height. The mortar properties are taken into account when joint stiffness and strength parameters are assigned. If a mortar thickness is actually plotted, then the system of Fig. 9 would appear somewhat more realistic, as shown in Fig. 11.

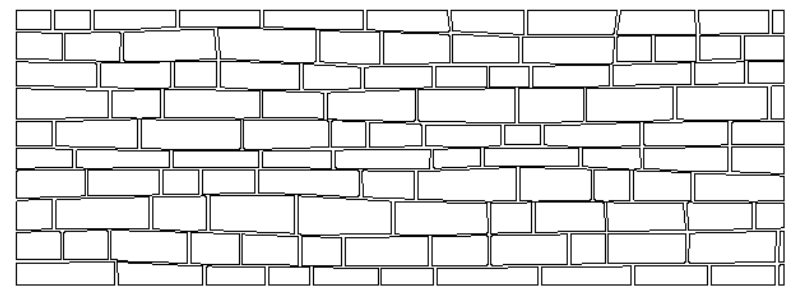

Fig. 11. Block plot displaying mortar thickness.

\section{NATURAL FREQUENCIES OF RIGID BLOCK SYSTEMS}

Rigid block models are primarily intended for failure analysis. However, there are often situations in which it is useful to analyze its elastic behavior, for example, to verify global deformability or characterize dynamic response. Rigid block codes usually employ explicit algorithms in the time domain, so they have no facilities to build stiffness matrices. The procedure employed in the example in the following section is briefly described here.

In 3D a rigid block has 6 degrees of freedom, 3 translations and 3 rotations. The stiffness matrix of the elastic rigid block system is defined in terms of these 6 
degrees of freedom per free block, and the corresponding forces and moments at the block centroids. The mechanical interaction between 2 blocks in 3DEC [9] is represented by means of a set of point contacts, which may be of 2 types, vertexto-face and edge-to-edge, as shown in Fig. 12.
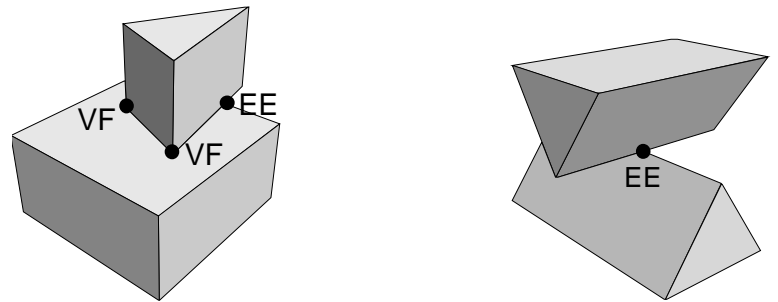

Fig. 12. Representation of block interaction by point contacts in 3DEC.

The contact normal, determined through Cundall's common plane algorithm, corresponds essentially to the normal to the block face or to the plane containing the 2 edges. Each point contact is assigned an area, which in the case of a face-toface interaction leads to the actual contact area. For a true point contact (as on the right of Fig. 12), a nominal area is assigned, which physically represents a minimal value of stiffness.

The stiffness matrix of a point contact, a $12 \times 12$ matrix, may be numerically built column by column, by prescribing small displacements and rotations of each block, one at a time, in the coordinate directions. The forces and moments originated by these configurations at the 2 block centroids constitute each matrix column. In this way, in an explicit code, the existing routines for the contact forcedisplacement laws are directly used to produce the elementary stiffness matrix. The stiffness matrices of all point contacts between a pair of blocks are added, and then these are assembled to get the global system matrix in the usual manner. Eigenvalues and eigenmodes may then be obtained, assuming a diagonal mass matrix.

In this procedure, linear elastic interactions are assumed, in terms of the normal and shear stiffness of joints or contacts. Therefore, the stiffness matrix and natural frequencies depend on the system connectivity. At different stages of the analysis, particular contacts may be separated thus not contributing to the system stiffness. At advanced stages of collapse, loose blocks will render the procedure unfeasible.

The accuracy obtained with rigid block models has been verified against continuum elastic solutions. Comparisons of frequencies and mode shapes for beam and plate bending problems have shown that the rigid block approximation is adequate [8]. For a good representation of the out-of-plane bending, it is recommended that three or more contact points across the thickness of the wall be used. 


\section{LOCAL MODELING OF WALL FAILURE}

The safety assessment of historical buildings usually involves two scales of numerical analysis: global and local. Global models are simplified, not only in terms of geometrical detail, but also in terms of material models, often linear elastic assumptions being adopted. The global dynamic behavior can be calibrated against in situ experiments. The local models are used to assess the safety of critical components, and need to represent the nonlinear behavior, whether pushover methods or dynamic analysis are used. Discrete element models are one of the tools available for this local modeling scale.

The local modeling of a structural component raises the problem of setting adequate boundary conditions, such that the effect of the surrounding structure is satisfactorily represented. In the example presented in this section (Fig. 13), elastic supports are used to provide the support of the wall at both ends, in the two horizontal directions. The stiffness of these elastic supports was calibrated so that the two lowest frequencies and mode shapes matched reasonably well the in situ measurements provided by ambient vibration tests.
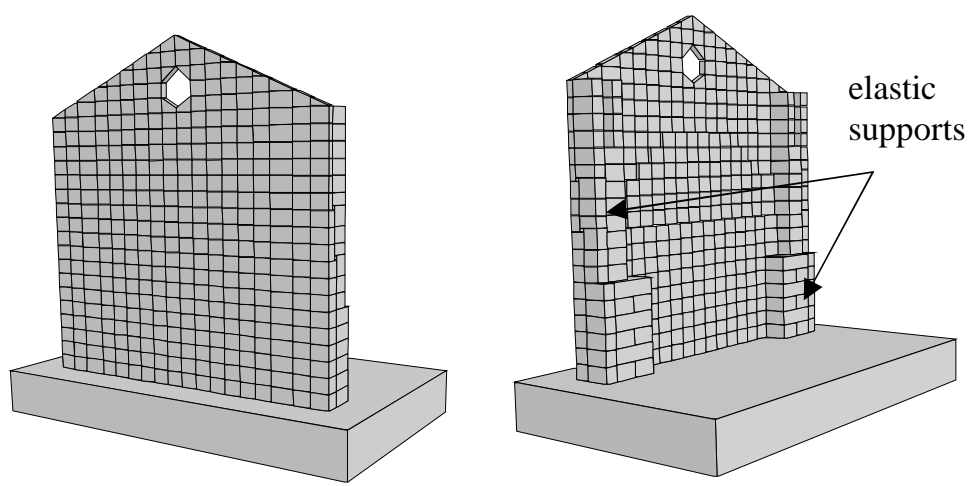

Fig. 13. Rigid block model for local wall failure analysis.

The wall shown in Fig. 13 has a maximum height of about $38 \mathrm{~m}$, at the centre, and a thickness varying thickness from $1.6 \mathrm{~m}$ at the base to $0.8 \mathrm{~m}$ at the top, with thicker buttresses near both ends. The rigid block model adopted a continuous joint pattern, which is a conservative assumption, according to the results discussed before. However, it should be noted that the numerical blocks are much larger than the actual blocks. Therefore, any sizeable offset might overestimate the effects of the actual interlocking, which may be taken into account through the cohesive strength of the vertical joints.

The wall modes were calculated in the assumption of elastic contacts, by the procedure outlined in the previous section. The first two modes are depicted in Fig. 14. This analysis was essential to calibrate the elastic supports on the sides of 
the model which represent the adjacent structure. The evaluation of the seismic capacity was based on pushover analyses $[12,13]$. Unlike the wall test problem, the seismic forces acting in the out-of-plane direction were not assumed uniform in height, but were applied to the rigid blocks according to the first mode shape, which dominates the response of the wall.
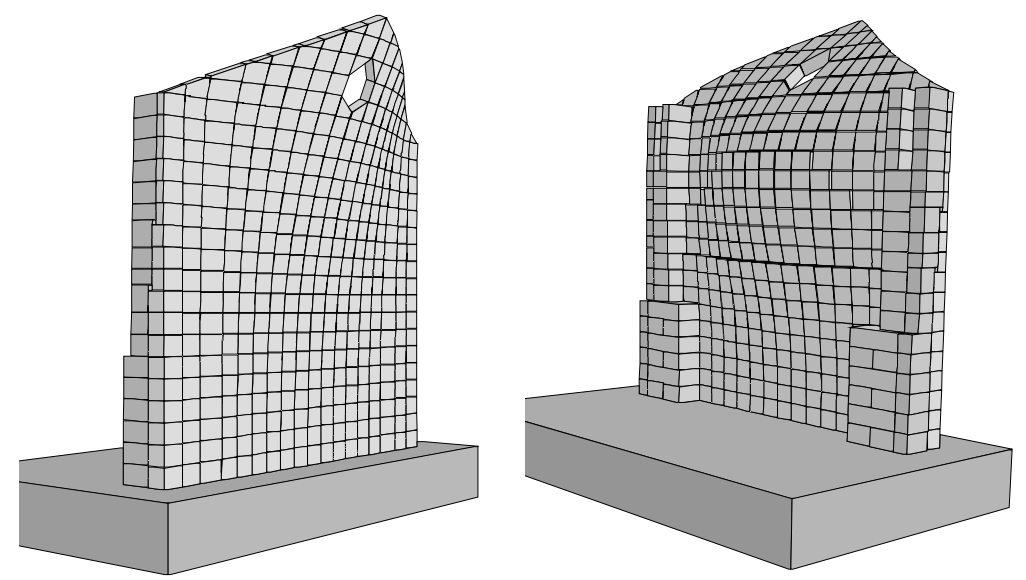

Fig. 14. First and second mode shapes of rigid block model.

In the nonlinear analyses, a Mohr-Coulomb model with cohesion and tensile strength was employed. Parametric studies were conducted to assess the influence of the main model parameters. As expected, failure is initiated by sliding and separation on the vertical joints near the thick lateral buttresses. The assumed continuity of these cross joints facilitates this mechanism, which is controlled by the assigned strength. The influence of the shear strength of the vertical joints on the wall capacity is plotted in Fig. 15. The 2 curves correspond to values of cohesion of 0.5 and $0.2 \mathrm{MPa}$, and friction angles of $35^{\circ}$ and $25^{\circ}$, respectively. In both cases, the joint tensile strength was $0.1 \mathrm{MPa}$. The lower strength case produces a markedly different behavior from the load level at which substantial shearing develops. 


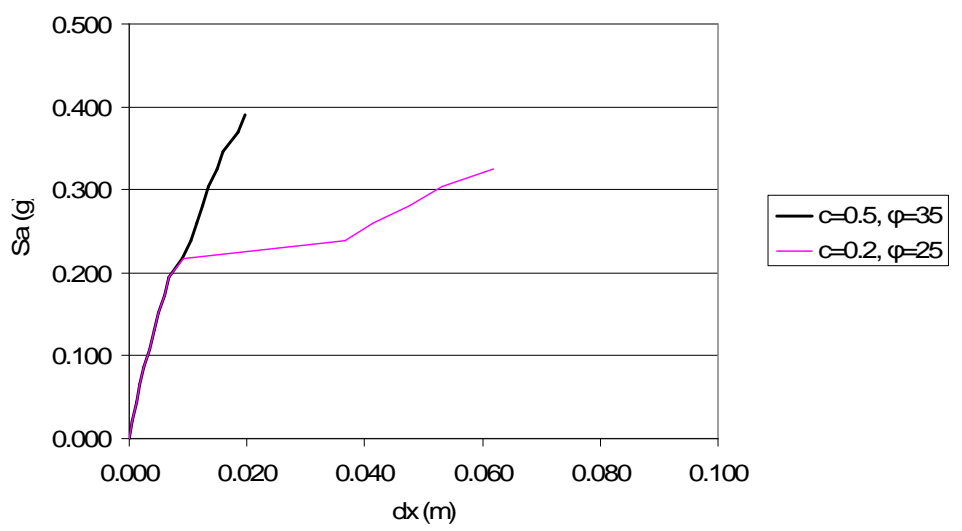

Fig. 15. Spectral acceleration vs. displacement curves for 2 values of vertical joint strength.

\section{CONCLUSIONS}

Discrete element models provide a powerful tool to analyze the deformation and failure modes of masonry, either in static or dynamic analysis. The successful application of these discontinuous representations involves judicious selection of model geometry and parameters. For simple stone structures, the model may reproduce the actual size and shape of individual blocks, so its generation is straightforward. For large and complex structures, however, the discrete block model is a considerable idealization. The effect of the simplified block patterns on the results needs to be assessed. For masonry walls under horizontal loads, it was shown that indiscriminate use of brick wall patterns with large cross joint offsets may overestimate significantly the wall strength. This is particularly important when the block size in the numerical model is larger than the real one, which is often unavoidable if the computational effort needs to be reduced.

For irregular masonry fabric, the use of random block generators, allows more realistic assemblies, avoiding the bias introduced by simple orthogonal joint sets. Voronoi polygons provide a simple option, but do not represent well the interlocking produced by staggered cross joints. In the test problem, these patterns led to wall strengths of the same order as the regular model with continuous cross joints. The alternative procedure proposed allows a more general class of masonry constructions to be addressed, and it provides a tool to evaluate the influence of irregularity and randomness, but it is still far from reproducing the real patterns. Further research on block generators capable of representing the various types of 
fabric found in historical masonry remains necessary for an effective application of discontinuous models.

Finally, it should be stressed that even if discontinuous models are mostly intended for collapse analysis, model checking and calibration under assumptions of elastic contact remains an important preliminary step for seismic studies.

\section{REFERENCES}

[1] Lemos JV (2007) Discrete element modeling of masonry structures. International Journal of Architectural Heritage, 1(2):190-213.

[2] Psycharis IN, Lemos JV, Papastamatiou DY, Zambas C, Papantonopoulos C (2003) Numerical study of the seismic behaviour of a part of the Parthenon Pronaos. Earthquake Engng Struct. Dyn., 32:2063-2084.

[3] Lourenço PB (2002) Computations of historical masonry constructions. Progress in Structural Engineering and Materials, 4:301-319.

[4] Casolo S, Pena F (2007) Rigid element model for in-plane dynamics of masonry walls considering hysteretic behaviour and damage. Earthquake Engng Struct. Dyn, 36:1029-1048.

[5] Potyondy DO, Cundall PA (2004) A bonded-particle model for rock. Int. J. Rock Mech. Min. Sciences, 41:1329-1364.

[6] Lemos JV (2006) Modeling of historical masonry with discrete elements. In: Mota Soares CA, Martins JAC, Rodrigues HC, Ambrósio JAC (eds.) III European Conf. on Computational Mechanics Solids, Structures and Coupled Problems in Engineering, Lisbon, June 5-8, 375-392.

[7] De Felice G, Giannini R (2001) Out-of-plane seismic resistance of masonry walls. Journal of Earthquake Engineering, 5(2):253-271.

[8] Lemos JV (2007) Numerical issues in the representation of masonry structural dynamics with discrete elements. In: M. Papadrakakis, D.C. Charmpis, N.D. Lagaros, Y. Tsompanakis (eds.) Compdyn2007, Rethymno, Crete, Greece, 13-16 June 2007, Paper 1126.

[9] 3DEC - Three-dimensional Distinct Element Code. Itasca Consulting Group, Minneapolis, 2006.

[10] Herbst M, Konietzky H, Walter K (2008) 3D microstructural modeling. In: Hart R, Detournay C, Cundall P (eds.) Continuum and Distinct Element Numerical Modeling in Geo-Engineering, Itasca Consulting Group, Minneapolis. 
[11] Pina-Henriques J, Lourenço PB (2006) Masonry compression: a numerical investigation at the meso-level. Engineering Computations, 23(4):382-407.

[12] Magenes G (2000) A method for pushover analysis in seismic assessment of masonry buildings. In: 12th World Conference on Earthquake Engineering, Auckland, New Zealand, paper 1866.

[13] Penna A (2008) Vulnerability assessment of masonry structures using experimental data and simplified models. Short Course on Post-Earthquake Buildings Safety and Damage Assessment, Eucentre, Pavia. 